\title{
Preditores e qualidade de vida em pacientes com radiodermatite: estudo longitudinal
}

\author{
Predictors and quality of life in patients with radiodermatitis: a longitudinal study \\ Predictores y calidad de vida en pacientes con radiodermatitis: estudio longitudinal
}

Daniel de Macêdo Rocha ${ }^{1}$ io https://orcid.org/0000-0003-1709-2143

Aliny de Oliveira Pedrosa ${ }^{1}$ io https://orcid.org/0000-0002-1396-9609

Aline Costa de Oliveira ${ }^{1}$ io https://orcid.org/0000-0003-1738-4808

Claudia Daniella Avelino Vasconcelos Benício ${ }^{1}$ io https://orcid.org/0000-0003-4638-2465

Ana Maria Ribeiro dos Santos ${ }^{1}$ id https://orcid.org/0000-0002-5825-5335

Lídya Tolstenko Nogueira ${ }^{1}$ io https://orcid.org/0000-0003-4918-6531

Como citar: CD, Santos AM, Nogueira LT. Preditores e qualidade de vida em pacientes com radiodermatite: estudo longitudinal. Acta Paul Enferm. 2021;34:eAPE01063.

DOI

http://dx.doi.org/10.37689/actaape/2021A001063

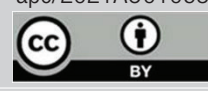

Descritores

Neoplasia; Radioterapia; Radiodermatite; Qualidade de vida

Keywords

Neoplasms; Radiotherapy; Radiodermatitis; Quality of life

Descriptores

Neoplasias; Radoterapía; Radiodematitis; Cualidad de vida

\section{Submetido 8 de Maio de 2020 \\ Aceito 20 de Agosto de 2020}

\section{Autor correspondente}

Daniel de Macêdo Rocha

E-mail: daniel_m.rocha@outlook.com.br

\section{Resumo}

Objetivo: Analisar os preditores sociodemográficos, clínicos e terapêuticos e a qualidade de vida em pacientes com radiodermatite.

Métodos: Trata-se de um estudo longitudinal, realizado com 196 pessoas que desenvolveram reações cutâneas como evento adverso ao tratamento radioterápico. Foi utilizado um formulário para caracterização e avaliação clínica e o instrumento European Organization for Research and Treatment of Cancer Quality of Life Questionnaire-Core30 para mensuração da qualidade de vida em duas etapas. A análise dos dados foi constituída pelos testes Wilcoxon, Mann-Whitney e Kruskal-Wallis, considerando diferença estatisticamente significativa para $p<0,05$.

Resultados: A comparação entre os escores de qualidade de vida mostrou que a radiodermatite contribuiu para piora do estado global de saúde e da qualidade de vida, deterioração da capacidade funcional, dificuldade financeira e intensificação de reações emocionais e de sintomas físicos como ansiedade, depressão, dor, fadiga, insônia e falta de apetite. Fatores como sexo, renda, escolaridade, quimioterapia concomitante, localização anatômica da lesão e grau de destruição tecidual foram determinantes para o maior comprometimento dos escores globais. Diante disso, surge a necessidade de intervenções de enfermagem que favoreçam a identificação de preditores e que otimizem o cuidado a partir de diretrizes e recomendações clínicas.

Conclusão: A radiodermatite influenciou negativamente a qualidade de vida dos pacientes, tendo alto impacto associado ao maior grau de destruição tecidual e como principais preditores destacaram-se os aspectos clínicos e terapêuticos. Estudos dessa natureza são imprescindíveis para formulação de políticas públicas efetivas, integradas, sustentáveis e baseadas em evidências voltadas para prevenção, controle e tratamento da lesão.

\section{Abstract}

Objective: To analyze sociodemographic, clinical and therapeutic predictors and quality of life in patients with radiodermatitis.

Methods: This longitudinal study was conducted with 196 participants who developed skin reactions as an adverse event to radiotherapy treatment. A form was used for clinical characterization and evaluation, and the European Organization for Research and Treatment of Cancer Quality of Life Questionnaire-Core30 instrument was used for measuring the quality of life in two stages. Data analysis consisted of the Wilcoxon, MannWhitney and Kruskal-Wallis tests, considering a statistically significant difference for $p<0.05$.

Results: The comparison between scores of the quality of life showed that radiodermatitis contributed to the worsening of overall health status and quality of life, deterioration of functional capacity, financial difficulty and intensification of emotional reactions and physical symptoms such as anxiety, depression, pain, fatigue, 
insomnia and appetite loss. Factors such as sex, income, educational level, concomitant chemotherapy, anatomical location of the injury and degre of tissue destruction were determinant for the greater impairment of global scores. Thus, the need for nursing interventions that favor the identification of predictors and care optimization based on clinical guidelines and recommendations.

Conclusion: Radiodermatitis negatively influenced the quality of life of patients, had a high impact associated with a greater degree of tissue destruction and the main predictors were clinical and therapeutic aspects. Studies of this nature are essential for the formulation of effective, integrated, sustainable and evidence-based public policies aimed at preventing, controlling and treating the injury.

\section{Resumen}

Objetivo: Analizar los predictores sociodemográficos, clínicos y terapéuticos y la calidad de vida en pacientes con radiodermatitis.

Métodos: Se trata de un estudio longitudinal, realizado con 196 personas que presentaron reacciones cutáneas como evento adverso al tratamiento radioterápico. Se utilizó un formulario para la caracterización y evaluación clínica y el instrumento European Organization for Research and Treatment of Cancer Quality of Life Questionnaire-Core30 para la medición de calidad de vida en dos etapas. El análisis de los datos se realizó a través de las pruebas Wilcoxon, Mann-Whitney y Kruskal-Wallis, considerando una diferencia estadísticamente significativa de $\mathrm{p}<0,05$.

Resultados: La comparación entre las puntuaciones de calidad de vida demostró que la radiodermatitis contribuyó con el empeoramiento del estado global de salud y de la calidad de vida, el deterioro de la capacidad funcional, la dificultad financiera y la intensificación de reacciones emocionales y de síntomas físicos como ansiedad, depresión, dolor, fatiga, insomnio y falta de apetito. Factores como sexo, ingresos, escolaridad, quimioterapia simultánea, ubicación anatómica de la lesión y nivel de destrucción tisular fueron determinantes para un mayor comprometimiento de la puntuación global. Ante esto, surge la necesidad de intervenciones de enfermería que favorezcan la identificación de predictores y que optimicen el cuidado a partir de directrices y recomendaciones clínicas.

Conclusión: La radiodermatitis influyó negativamente en la calidad de vida de los pacientes, con un alto impacto relacionado con un mayor nivel de destrucción tisular, y como principales predictores se destacaron los aspectos clínicos y terapéuticos. Estudios de esta naturaleza son imprescindibles para la formulación de políticas públicas efectivas, integradas, sustentables y basadas en evidencias orientadas hacia la prevención, control y tratamiento de la lesión.

\section{Introdução}

Apesar dos avanços terapêuticos e do crescente interesse para o gerenciamento de lesóes de pele, a radiodermatite se configura como principal evento adverso da terapia radioterápica, constituindo um grave problema por apresentar elevada incidência, comprometer a Qualidade de Vida (QV) dos pacientes, gerar custos elevados para os serviços de saúde e requerer esforços substanciais para prevenção, controle e tratamento. ${ }^{(1,2)}$

A radiodermatite compreende um conjunto de lesões cutâneas decorrente da exposição intensa a radiação ionizante que acomete $85 \%$ dos pacientes, podendo ser intensificadas por condiçôes físicas, clínicas e terapêuticas, uma vez que a extensão da área irradiada, terapias concomitantes e os hábitos de vida diária podem contribuir para o maior grau de destruição tecidual. ${ }^{(3,4)}$

Embora as alteraçóes iniciem após a primeira radiação, a exposição contínua à doses fracionadas acarreta danos celulares contínuos, que impedem a reparação tecidual e que intensificam após a segunda semana de tratamento com a manifestação de eritema leve, descamação seca ou úmida, extravasamento de fluidos, necrose, ulceraçôes profundas e infecção local. ${ }^{(5,6)}$

São incipientes as diretrizes clínicas e os protocolos assistenciais que descrevam os cuidados de enfermagem ou os produtos tópicos adequados para manejo da reação. No entanto, a prevenção é reco- mendada, podendo ser direcionada pela experiência clínica do enfermeiro, grau de destruição tecidual, disponibilidade de recursos materiais, uso de produtos tópicos e orientaçóes de cuidados com a pele. ${ }^{(7)}$

A literatura demonstra que a magnitude da reação pode ser mensurada pelas incapacidades e pelos comprometimentos na QV..$^{(2,7)}$ Assim, o impacto associado à lesão pode refletir a insatisfação com a eficácia terapêutica, levando a limitaçôes físicas, ao desenvolvimento de reaçôes emocionais, a intensificação de sintomas e ao prolongamento ou interrupção da progressão do tratamento. ${ }^{(8,9)}$

No campo da oncologia a mensuração da QV é amplamente referenciada, visando direcionar políticas públicas, assim como nortear a prática assistencial, a definição de condutas, o planejamento do processo de reabilitação e a avaliação dos cuidados, considerando a subjetividade das pessoas por meio de ferramentas e instrumentos de medida. ${ }^{(9,10)}$

Diferentes concepçóes são aplicadas ao termo "Qualidade de Vida", que envolvem parâmetros sociais, econômicos e de saúde. Neste estudo foi adotada a definição proposta pela Organização Mundial de Saúde que considera a "percepção do indivíduo acerca de sua posição na vida, de acordo com o contexto cultural e sistema de valor com os quais convive e em relação a seus objetivos, expectativas, padrōes e preocupaçóes" ${ }^{\text {(11) }}$ por entender que essa 
conceituação contempla todo o percurso terapêutico que o paciente oncológico possa ser submetido.

Considerando a possibilidade de minimizar os impactos relacionados à radiodermatite a partir da mensuração da QV e de abordagens direcionadas às suas características, o objetivo deste estudo foi analisar os preditores sociodemográficos, clínicos e terapêuticos e a qualidade de vida em pacientes com radiodermatite.

\section{Métodos}

Trata-se de um estudo observacional longitudinal, realizado na divisão de radioterapia de uma instituição hospitalar referência para tratamento oncológico de Teresina, Piauí, Brasil, no período de janeiro a julho de 2018.

O delineamento da amostra foi realizado por meio da técnica para populaçóes infinitas, considerando a prevalência presumida da radiodermatite de $85 \%$, margem de erro de $5 \%$ e nível de confiança de 95\%. Assim, o estudo foi constituído por 196 pessoas em terapia radioterápica, com idade igual ou superior a 18 anos e prescrição mínima de 12 sessóes. Foram excluídos os pacientes com complicaçóes neurológicas, clínicas ou cognitivas, incapacidades para compreensão dos formulários e os que não apresentaram reaçóes cutâneas durante o tratamento.

Para caracterização sociodemográfica, clínica e terapêutica, assim como para avaliação da presença e da intensidade da toxicidade cutânea, foram elaborados dois formulários após revisão de literatura ${ }^{(2)}$ e seleção das melhores evidências, sendo submetido à apreciação de especialistas para avaliação quanto a pertinência, objetividade e clareza dos itens, validação de conteúdo e adequação aos objetivos propostos. Nessa perspectiva, as variáveis investigadas foram sexo, idade, estado civil, escolaridade, religiáo, procedência, comorbidades prévias, localização do câncer, tempo de diagnóstico e de tratamento, número de sessóes radioterápicas prescritas, grau de destruição tecidual e terapias concomitantes.

Destaca-se que para classificação dos graus de toxicidade foram seguidas as recomendações propostas pelo Radiation Therapy Oncology Group (RTOG), que classifica a reação em cinco níveis de destruição tecidual: grau 0 - sem reação, pele ínte- gra; grau 1 - eritema leve, epilação e/ou descamação seca; grau 2 - eritema doloroso, descamação úmida localizada e/ou edema moderado; grau 3 - descamação úmida confluente e/ou edema importante; e grau 4 - ulceração, hemorragia e/ou necrose. ${ }^{(12)}$

A mensuração da qualidade de vida foi realizada por meio do instrumento European Organization for Research and Treatment of Cancer Quality of Life Questionnaire-Core30 (EORTC QLQ-C30). Trata-se de uma ferramenta desenvolvida pela European Organization Research Treatment of Cancer (EORTC), traduzida e validada para a língua portuguesa, sendo composta por 30 itens distribuídos em quatro escalas e quinze domínios que avaliam o estado de saúde global e qualidade de vida, a capacidade funcional, a presença e intensidade de sintomas e de dificuldades financeiras. ${ }^{(13)}$

Todos os escores foram padronizados em uma transformação linear que varia de 0 a 100, conforme recomendaçôes propostas pelo Scoring Manual da EORTC, ${ }^{(14)}$ em que para o estado de saúde global e escalas funcionais, quanto maior a pontuação, melhor a QV. Já em relação à escala de sintomas, os escores elevados refletem maior intensidade de sintomatologias e pior QV. Destaca-se que para utilização deste instrumento, foi solicitada e obtida autorização por meio do registro do projeto de pesquisa.

Os dados foram coletados em duas etapas, após contato com o enfermeiro responsável pelo setor de radioterapia para levantamento dos pacientes que atendiam aos critérios de inclusão, certificação do diagnóstico, do tratamento e seleção da amostra. Considerando a baixa escolaridade comum na população estudada, optou-se pela entrevista, em que a leitura dos itens foi realizada pelo pesquisador, com tempo médio para avaliação de 30 minutos.

A primeira etapa compreendeu o período inicial do tratamento radioterápico, predominantemente até a décima sessão, sendo coletadas variáveis sociodemográficas, clínicas e terapêuticas mediante análise de prontuário e aplicado o instrumento EORTC QLQ-C30 para mensuração da QV antes da manifestação do evento adverso. A segunda fase também ocorreu durante a radioterapia, após a décima segunda aplicação, especificamente no momento correspondente ao pior estágio da lesão, sendo realizada a ava- 
liação da intensidade e das características da reação e aplicado o EORTC QLQ-C30 para avaliação da QV.

A inserção dos participantes na pesquisa aconteceu de forma aleatória e durante o estudo 234 pessoas eram potencialmente elegíveis. Destes 38 foram excluídos, sendo 15 na primeira etapa por incapacidades clínicas, neurológicas e cognitivas e 23 na segunda por não apresentar reaçóes cutâneas no período de avaliação. Assim, 196 pessoas em tratamento radioterápico participaram das duas etapas da pesquisa.

Os dados foram inseridos em planilha com dupla entrada no software da Microsoft Office Excel e exportados para o programa Statistical Package for the Social Sciences (SPSS). As variáveis sociodemográficas, clínicas e terapêuticas foram expressas em média, desvio padrão, máximo e mínimo, frequência absoluta e relativa.

$\mathrm{O}$ teste de aderência de Kolmogorov-Smirnov foi realizado para verificação da distribuição dos dados, Wilcoxon para comparação entre os escores médios de QV antes e após o desenvolvimento da lesão, Mann-Whitney e Kruskal-Wallis para verificar associações entre a QV e as variáveis qualitativas dicotômicas e politômicas, respectivamente. Todas as análises foram conduzidas ao nível de significância de $5 \%$, para resultados com p valor abaixo de 0,05 , sendo considerados significantes $(\mathrm{p}<0,05)$.

O estudo foi aprovado pelo Comitê de Ética em Pesquisa da Universidade Federal do Piauí e o parecer favorável foi emitido por meio do protocolo número 2.379.708. A participação foi voluntária e condicionada à assinatura do Termo de Consentimento Livre e Esclarecido.

\section{Resultados}

O estudo descritivo da amostra evidenciou o predomínio do sexo feminino 126 (64,3\%), com ida-

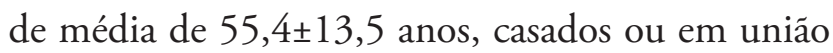
estável 125 (63,8\%), aposentados 78 (39,8\%), católicos $152(77,6 \%)$, com ensino fundamental $101(51,5 \%)$, de baixa renda $116(59,2 \%)$ e procedentes de outros municípios do Piauí 89 (45,4\%). Quanto às condiçôes clínicas, os participantes, em sua totalidade, apresentaram comorbidades e fatores associados para o desenvolvimento do câncer, sendo prevalente o histórico familiar da doença 112 $(57,1 \%)$, seguido do tabagismo $83(42,4 \%)$, da hipertensão arterial sistêmica $61(31,1 \%)$, do etilismo $46(23,5 \%)$ e da diabetes mellitus 28 (14,3\%).

O tempo de diagnóstico variou de 3 a 6 meses para a maioria das pessoas $132(67,4 \%)$ e as localizaçóes anatômicas mais acometidas pela neoplasia foram a mama $52(26,5 \%)$, a cabeça e pescoço $40(20,4 \%)$, a próstata 39 (19,9\%) e o colo do útero 38 (19,4\%). Em relação aos métodos terapêuticos, prevaleceram prescriçóes entre 12 a 30 sessóes radioterápicas 153 $(78,1 \%)$ e a administração concomitante de quimioterapia foi adotada para $70(35,7 \%)$ participantes.

A avaliação das características clínicas da radiodermatite, mostrou a prevalência de reaçôes grau II em 115 (58,7\%) pessoas, com tecido de epitelização 118 (60,2\%), sem exsudato 144 (73,5\%) e com maior incidência na região inguinal $73(37,2 \%)$, mama 54 (27,6\%), e cabeça e pescoço 39 (19,9\%). Os métodos terapêuticos adotados para controle e tratamento da lesão basearam-se na aplicação tópica da compressa com camomila 189 (96,4\%), do creme de aloe vera 182 (92,9\%), do Ácido Graxo Essencial (AGE) 19 (9,7\%), do hidrogel $5(2,6 \%)$ e da sulfadiazina com prata $2(1,0 \%)$.

Os resultados expressos na tabela 1 demonstram a comparação entre escores médios de QV mensurados nas duas etapas do estudo. Observa-se na escala saúde

Tabela 1. Escores médios de qualidade de vida antes e depois da radiodermatite

\begin{tabular}{lccc}
\hline Escalas e domínios & $\begin{array}{c}\text { Primeira avaliação } \\
\mathbf{M} \pm \mathbf{D P}\end{array}$ & $\begin{array}{c}\text { Segunda avaliação } \\
\mathbf{M} \pm \mathbf{D P}\end{array}$ & p-value \\
\hline Saúde global e QV & $76,5 \pm 15,7$ & $35,5 \pm 18,5$ & $<0,001^{*}$ \\
Funções & & & \\
$\quad$ Física & $84,8 \pm 20,4$ & $65,8 \pm 25,3$ & $<0,001^{*}$ \\
Desempenho de papéis & $77,9 \pm 24,7$ & $48.9 \pm 31,0$ & $<0,001^{*}$ \\
Emocional & $77,8 \pm 21,4$ & $41,8 \pm 29,56$ & $<0,001^{*}$ \\
Cognitiva & $89,2 \pm 16,0$ & $82,6 \pm 23,5$ & $<0,001^{*}$ \\
Social & $85,9 \pm 18,2$ & $58,4 \pm 28,3$ & $<0,001^{*}$ \\
Sintomas & & & \\
Fadiga & $19,1 \pm 16,7$ & $50,9 \pm 22,9$ & $<0,001^{*}$ \\
Náusea e vômitos & $10,5 \pm 18,0$ & $18,0 \pm 27,8$ & $<0,001^{*}$ \\
Dor & $5,1 \pm 11,3$ & $40,1 \pm 25,1$ & $<0,001^{*}$ \\
Dispneia & $3.4 \pm 11,6$ & $4,5 \pm 12,8$ & 0,108 \\
Insônia & $21,9 \pm 27,7$ & $54,1 \pm 35,3$ & $<0,001^{*}$ \\
Falta de apetite & $16,2 \pm 24,3$ & $40,8 \pm 38,6$ & $<0,001^{*}$ \\
Constipação & $11,0 \pm 22,9$ & $9,6 \pm 18,7$ & 0,401 \\
Diarreia & $8,8 \pm 21,3$ & $7,2 \pm 14,5$ & 0,257 \\
Dificuldade financeira & $51,8 \pm 37,6$ & $80,6 \pm 30,5$ & $<0,001^{*}$ \\
\hline
\end{tabular}

M ( \pm DP) - média e desvio padrão; *Diferença significativa para $p<0,05$ 
global e QV que após o aparecimento da reação houve redução dos escores, com diferenças significativas $(\mathrm{p}<$ $0,05)$, assim como na escala de funçóes que apresentou maior comprometimento dos domínios físico, desempenho de papéis, emocional e social. Nos sintomas, houve o aumento das pontuaçóes nos domínios fadiga, náuseas e vômitos, dor, insônia e falta de apetite, indicando, assim, a intensificação da sintomatologia.

A tabela 2 apresenta a comparação entre as características sociodemográficas e terapêuticas com as escalas e domínios do EORTC QLQ C-30. Dentre as associaçóes, verificou-se que os escores das escalas saúde global e QV, funçáo, sintomas e dificuldade financeira foram determinados por diferentes aspectos, tais como escolaridade, renda, tempo de tratamento e quimioterapia concomitante $(\mathrm{p}<0,05)$.

A localização anatômica da lesão, especificamente em mama e regiáo inguinal, assim como o grau da reaçáo e o tipo de tecido representaram importantes preditores clínicos associados ao pior estado global de saúde e qualidade de vida (Tabela 3).

Tabela 2. Análise dos preditores sociodemográficos e terapêuticos para qualidade de vida em pacientes com radiodermatite

\begin{tabular}{|c|c|c|c|c|c|c|c|}
\hline Escalas e domínios / Variáveis & $\begin{array}{l}\text { Sexo } \\
p \text {-value }\end{array}$ & $\begin{array}{c}\text { Estado civil } \\
p \text {-value }\end{array}$ & $\begin{array}{c}\text { Escolaridade } \\
p \text {-value }\end{array}$ & $\begin{array}{l}\text { Renda } \\
p \text {-value }\end{array}$ & $\begin{array}{l}\text { Localização do } \\
\text { câncer } \\
p \text {-value }\end{array}$ & $\begin{array}{c}\mathrm{N}^{\circ} \text { de sessões } \\
p \text {-value }\end{array}$ & $\begin{array}{c}\text { Quimioterapia } \\
\text { p-value }\end{array}$ \\
\hline Saúde global e QV & 0,095 & 0,793 & 0,489 & 0,522 & 0,106 & 0,652 & $0,002^{*}$ \\
\hline \multicolumn{8}{|l|}{ Funções } \\
\hline Função física & 0,878 & 0,706 & 0,964 & 0,412 & $0,015^{*}$ & $0,036^{*}$ & $0,026^{\star}$ \\
\hline Desempenho de papéis & $0,008^{\star}$ & $0,046^{*}$ & 0,732 & 0,152 & 0,074 & 0,440 & $0,003^{*}$ \\
\hline Função emocional & $0,010^{\star}$ & 0,951 & 0,061 & 0,421 & 0,07 & 0,307 & 0,133 \\
\hline Função cognitiva & 0,906 & 0,157 & $0,018^{*}$ & 0,151 & 0,549 & 0,129 & 0,678 \\
\hline Função social & 0,584 & $0,023^{*}$ & 0,620 & 0,331 & 0,437 & 0,291 & 0,118 \\
\hline \multicolumn{8}{|l|}{ Sintomas } \\
\hline Fadiga & $0,018^{\star}$ & 0,607 & 0,526 & $0,008^{*}$ & $0,026^{*}$ & 0,356 & $0,001^{*}$ \\
\hline Náusea e vômitos & 0,191 & 0,506 & 0,420 & 0,586 & $0,01^{*}$ & 0,763 & $0,001^{*}$ \\
\hline Dor & 0,609 & 0,664 & 0,299 & 0,401 & 0,488 & 0,494 & $0,001^{*}$ \\
\hline Dispneia & 0,127 & 0,212 & 0,995 & 0,967 & 0,195 & $0,005^{*}$ & $0,016^{*}$ \\
\hline Insônia & $0,018^{*}$ & 0,563 & 0,648 & 0,517 & 0,210 & 0,182 & 0,138 \\
\hline Falta de apetite & 0,146 & 0,172 & 0,854 & 0,392 & $0,025^{\star}$ & 0,198 & $0,001^{*}$ \\
\hline Constipação & 0,677 & $0,001^{*}$ & 0,568 & 0,701 & $0,014^{*}$ & 0,128 & 0,285 \\
\hline Diarreia & 0,083 & 0,119 & 0,873 & 0,774 & $0,001^{*}$ & 0,18 & 0,19 \\
\hline Dificuldade financeira & 0,133 & 0,716 & $0,033^{*}$ & $0,001^{*}$ & 0,669 & 0,229 & 0,645 \\
\hline
\end{tabular}

*Diferença significativa para $\mathrm{p}<0,05$

Tabela 3. Preditores clínicos da qualidade de vida de pacientes com radiodermatite

\begin{tabular}{|c|c|c|c|c|c|c|c|}
\hline $\begin{array}{l}\text { Escalas e domínios/ localização e características } \\
\text { clinicas }\end{array}$ & $\begin{array}{c}\text { Cabeça e } \\
\text { pescoço } \\
p \text {-value }\end{array}$ & $\begin{array}{l}\text { Mama } \\
p \text {-value }\end{array}$ & $\begin{array}{l}\text { Vaginal } \\
p \text {-value }\end{array}$ & $\begin{array}{l}\text { Inguinal } \\
p \text {-value }\end{array}$ & $\begin{array}{c}\text { Grau } \\
p \text {-value }\end{array}$ & $\begin{array}{c}\text { Tipo de tecido } \\
\text { p-value }\end{array}$ & $\begin{array}{c}\text { Quantidade de } \\
\text { exsudato } \\
p \text {-value }\end{array}$ \\
\hline Saúde global e QV & $0,035^{\star}$ & 0,180 & 0,134 & $0,045^{*}$ & $<0,001^{\star}$ & $<0,001^{*}$ & $<0,001^{*}$ \\
\hline \multicolumn{8}{|l|}{ Funções } \\
\hline Função física & 0,957 & $0,017^{\star}$ & 0,831 & $0,018^{\star}$ & $<0,001^{*}$ & $<0,001^{*}$ & $0,007^{\star}$ \\
\hline Desempenho de papéis & 0,144 & 0,188 & 0,886 & $0,003^{*}$ & $<0,001^{\star}$ & $<0,001^{*}$ & $0,002^{*}$ \\
\hline Função emocional & 0,366 & 0,199 & 0,805 & 0,807 & 0,759 & 0,892 & 0,508 \\
\hline Função cognitiva & 0,111 & 0,825 & 0,903 & 0,340 & $0,002^{*}$ & $0,046^{\star}$ & $<0,001^{*}$ \\
\hline Função social & $0,020^{*}$ & 0,504 & 0,942 & $0,003^{*}$ & $<0,001^{*}$ & $<0,001^{*}$ & $<0,001^{*}$ \\
\hline \multicolumn{8}{|l|}{ Sintomas } \\
\hline Fadiga & 0,065 & 0,191 & 0,063 & 0,726 & $0,020^{\star}$ & $0,020^{*}$ & 0,259 \\
\hline Náusea e vômitos & $0,03^{*}$ & 0,947 & 0,105 & 0,671 & $<0,001^{*}$ & $<0,001^{*}$ & $<0,001^{*}$ \\
\hline Dor & 0,538 & 0,412 & 0,793 & 0,173 & 0,381 & 0,550 & 0,391 \\
\hline Dispneia & 0,392 & 0,225 & 0,817 & 0,141 & $0,022^{*}$ & $0,013^{\star}$ & $0,016^{\star}$ \\
\hline Insônia & 0,108 & 0,602 & $0,039^{*}$ & $0,032^{*}$ & $0,001^{\star}$ & $<0,001^{*}$ & 0,070 \\
\hline Falta de apetite & 0,518 & 0,251 & 0,361 & 0,996 & $0,039^{*}$ & 0,183 & 0,350 \\
\hline Constipação & 0,066 & 0,584 & 0,454 & 0,938 & 0,553 & 0,871 & 0,116 \\
\hline Diarreia & $0,036^{*}$ & 0,754 & $0,006^{\star}$ & 0,116 & $<0,001^{*}$ & $<0,001^{*}$ & $<0,001^{*}$ \\
\hline Dificuldade financeira & 0,997 & 0,083 & 0,799 & 0,182 & $0,001^{*}$ & $<0,001^{\star}$ & $0,002^{\star}$ \\
\hline
\end{tabular}




\section{Discussão}

Os resultados deste estudo corroboram com o perfil epidemiológico da lesão em âmbito nacional e internacional, em que o maior acometimento da regiáo inguinal, mama e cabeça e pescoço são evidenciados. A elevada incidência da radiodermatite nessas regióes está relacionada ao campo de tratamento, uma vez que áreas extensas, com incidência direta da radiaçáo, pouco tecido adiposo ou presença de dobras tornam-se comuns umidade e fricção, resultando na fragilidade cutânea. ${ }^{(15)}$

As manifestaçōes da lesão ocorreram, em sua totalidade, após a segunda semana de tratamento, mantendo-se em grau II, assim como em outra pesquisa que apontou a presença da lesão a partir da $12^{\mathrm{a}}$ sessão radioterápica. A gravidade das reações pode ser atribuída ao plano terapêutico, em que a técnica e o elevado número de sessóes é determinante para o maior grau de destruição tecidual, uma vez que a radiação contínua impede as fases de proliferação, maturação e reparação comuns no processo de cicatrização. ${ }^{(5,16)}$

Apesar de serem incipientes as recomendaçóes clínicas e evidências de validade, segurança e eficácia comprovada, a aplicação de compressas a base de camomila e cremes de aloe vera foi frequente, devido às atividades radioprotetoras, efeitos anti-inflamatórios e analgésicos já evidenciadas, assim como o seu potencial para controlar sintomas físicos e retardar a ocorrência da lesão. ${ }^{(17,18)}$

Nesse sentido, o cuidado liderado pelo enfermeiro torna-se fundamental e se destaca por envolver no gerenciamento da lesão atividades voltadas para avaliaçâa adequada do grau toxicidade, no controle de comportamentos e hábitos de vida que dificultam a reparação tecidual, no reconhecimento de preditores que interferem na condução, adesão ao tratamento e sobrevida dos pacientes, assim como no atendimento às necessidades de informação, promoção do autocuidado e manutençáo da qualidade de vida. . $^{(16-19)}$

A mensuração inicial da QV mostrou menores comprometimentos dos escores globais, evidenciando melhor estado de saúde/QV, assim como menor intensidade de sintomas físicos e de dificuldades financeiras. Esses comprometimentos podem refletir a ausência de eventos adversos à modalidade tera- pêutica, o processo de aceitação e autoconfiança, os mecanismos para enfrentamento da doença e a capacidade para adaptação à nova condição de vida, uma vez que o processo de cura que é iniciado. ${ }^{(20)}$

Apesar de considerar que outras condiçôes clínicas podem provocar impacto na vida dos pacientes, a literatura evidencia que a $\mathrm{QV}$ diminui durante a radioterapia devido a elevada incidência de eventos adversas como as dermatites, levando à incapacidade, complicações e comorbidades psicopatológicas. ${ }^{(2)}$ Assim, este estudo sugere que a avaliaçáo da QV deve ser realizada para que os cuidados de suporte se concentrem nos prejuízos identificados.

As avaliaçôes realizadas após a identificação do pior estágio da radiodermatite mostraram impactos negativos, indicando maior comprometimento das escalas de funções, especificamente nos domínios físico, funçáo emocional, cognitiva e social, bem como intensificação de sintomas clínicos.

Dentre as escalas e domínios, a dificuldade financeira foi a mais acometida, apresentando elevação do escore médio para 80,61 $\pm 30,47$ e corroborando com as características dos participantes, uma vez que a maioria era aposentada ou desempregada e que se deslocavam do interior ou de outros estados em virtude da centralização de instituiçóes de referência, resultando na desestruturação e distanciamento familiar e aumento de custos. Além disso, o grau de destruição tecidual contribuiu para maior comprometimento desse escore, tendo em vista a necessidade da aquisiçâo de recursos terapêuticos e produtos tópicos para o seu gerenciamento. ${ }^{(21)}$

A dificuldade financeira pode refletir ainda a realidade brasileira no segmento social, laboral e econômi$\mathrm{co}$, em que as incapacidades decorrentes da doença e do tratamento são relacionadas a perda da produtividade, as dificuldades no acesso à informação e as deficiências no reconhecimento de fatores de risco, acarretando diagnósticos tardios, maior grau de dependência e elevaçáo dos indicadores de mortalidade. ${ }^{(22)}$

Na literatura, a piora do estado global de saúde e da $\mathrm{QV}$ relacionada à radiodermatite também é relatada, em que o tempo elevado de tratamento, a extensão da área irradiada e a grau de destruição tecidual são preditores para limitação da capacidade funcional, intensificação de sintomas físicos e de reaçōes emocionais. ${ }^{(1,7)}$ 
Consistente com evidências internacionais, a quimioterapia concomitante ao tratamento radioterápico repercutiu nos escores médios e representou fator de risco para o desenvolvimento da reação, resultando em maior extensão da lesão e no acometimento de estruturas secundárias como a região axilar, comum em casos de neoplasias mamárias. ${ }^{(23,24)}$ Ensaios clínicos realizados constataram maior incidência da reação em pacientes submetidos à quimioterapia como coadjuvante ao plano terapêutico. ${ }^{(25,26)}$

Para a escala de funçôes, o maior comprometimento se concentrou no domínio emocional, revelando impacto significativo no bem-estar psicológico. Caracterizada pelo estresse e pela diminuição da capacidade física, variáveis clínicas e terapêuticas como o número de sessóes radioterápicas, quimioterapia concomitante e grau da lesão associaram-se e resultaram na alteração nas prioridades da vida, preocupaçóes constantes, sintomas de ansiedade e depressão, incertezas e baixa autoestima. ${ }^{(27)}$

Os domínios desempenho de papeis e função física também foram acometidos e contribuíram para pior QV. A variável idade esteve associada, mostrando que o aumento da expectativa de vida favorece a incidência de doenças crônicas não transmissíveis que representam demanda expressiva para os serviços de saúde e que se configuram como causas de hospitalização entre adultos mais velhos. ${ }^{(28,29)}$

Escores da escala função social relacionaram-se ao estado civil, grau da lesão e acometimento da cabeça e pescoço. A lesão quando desenvolvida em localizaçóes anatômicas específicas pode acarretar alteraçôes da imagem corporal que potencializam os riscos para isolamento social e para repercussóes emocionais, refletindo a necessidade do fortalecimento de redes de apoio e suporte, assim como a participação ativa dos familiares no plano de cuidados. ${ }^{(30)}$

$\mathrm{Na}$ escala de sintomas a comparação dos escores demonstrou maior intensidade de fadiga, náuseas e vômitos, dor, insônia e falta de apetite. Reconhecidas como sintomatologias comuns nos pacientes oncológicos, esses agravos estiveram atribuídos a incidência de reaçóes adversas como o alto grau de destruição tecidual que causa repercussóes no estado global de saúde. ${ }^{(7,18)}$
Assim, a dor de alta intensidade, sintoma mais reportado, gerou maior impacto na qualidade de vida e também foi verificada em outras pesquisas. ${ }^{(31,32)}$ Níveis elevados de dor constitui uma condição frequente e de grande expressão em pacientes com radiodermatite, tornando-se acentuada conforme o maior grau de destruição tecidual, limitando as atividades de vida diária e a capacidade funcional, e gerando desconforto psicológico e estados de dependência. ${ }^{(18)}$

Apesar da alta prevalência da dor, um dos desafios é o subdiagnóstico relacionado à estratégias ineficazes para avaliação, dificuldade ou relutância em expressar sintomas, preocupação relacionada à dependência de drogas analgésicas ou receio de reaçôes e interaçóes medicamentosas. ${ }^{(33)}$

A fadiga e as alteraçóes no padrão de sono também foram comuns, sendo caracterizada pela sensação subjetiva de cansaço decorrente do desgaste preexistente, essas manifestaçôes reduzem a funcionalidade e independência, sendo intensificadas quando combinadas com reaçóes emocionais e níveis elevados de dor. ${ }^{(34,35)}$

Diante da magnitude da reação e dos impactos na QV, surge a necessidade de intervençôes de enfermagem efetivas, como a identificação dos preditores e a implantação de estratégias voltadas para prevenção, controle e tratamento da lesão. Ainda, considera-se que conhecer a QV desses pacientes permite que o planejamento da assistência seja realizado a partir de diretrizes e recomendaçóes clínicas, voltadas para cuidado seguro.

As limitaçóes da pesquisa referem-se ao delineamento que não permite estabelecer relação de causa e efeito, assim como as falhas dos registros em prontuários dos pacientes, o que dificultou a identificação de variáveis relacionadas à radiação.

\section{Conclusão}

A radiodermatite gerou impactos negativos no estado global de saúde e na QV dos pacientes, apresentando maiores comprometimentos na capacidade funcional, desempenho de papéis, função física, social e emocional. Além disso, gerou dificuldades financeiras e intensificou sintomatologias como fa- 
diga, náuseas e vômitos, dor e insônia. Variáveis sociodemográficas, clínicas e terapêuticas como sexo, escolaridade, renda, tempo de tratamento, número de sessóes radioterápicas, quimioterapia concomitante, localização da reação e grau de destruição tecidual foram preditoras para pior QV.

\section{Colaborações}

Rocha DM, Pedrosa AO e Oliveira AC contribuíram com a concepção do projeto, análise e interpretação dos dados e redação do artigo. Santos AMR, Benício CDAV, Nogueira LT contribuíram para revisão crítica do conteúdo intelectual e aprovação final da versão a ser publicada.

\section{Referências}

1. Beamer LC, Grant M. Longitudinal trends in skin-related and global quality of life among women with breast radiodermatitis: A pilot study. Eur J Oncol Nurs. 2018;33:22-7.

2. Rocha DM, Pedrosa AO, Oliveira AC, Bezerra SM, Benício CD, Nogueira LT. Scientific evidence on factors associated with the quality of life of radiodermatitis patients. Rev Gaúcha Enferm. 2018;39(e2017-0224):1-8.

3. Bray FN, Simmons BJ, Wolfson AH, Nouri K. Acute and chronic cutaneous reactions to ionizing radiation therapy. Dermatol Ther (Heidelb). 2016;6(2):185-206.

4. Ryan JL. Ionizing radiation: the good, the bad, and the ugly. J Invest Dermatol. 2012;132(3 Pt 2):985-93.

5. Schneider F, Danski MT, Vayego SA. Usage of Calendula officinalis in the prevention and treatment of radiodermatitis: a randomized doubleblind controlled clinical trial. Rev Esc Enferm USP. 2015;49(2):221-8.

6. Huang A, Glick SA. Genetic susceptibility to cutaneous radiation injury. Arch Dermatol Res. 2017;309(1):1-10.

7. Fuzissaki MA, Paiva CE, Oliveira MA, Lajolo Canto PP, Paiva Maia YC. The impact of radiodermatitis on breast cancer patients' quality of life during radiotherapy: a prospective cohort study. J Pain Symptom Manage. 2019;58(1):92-99.e1.

8. Spałek M. Chronic radiation-induced dermatitis: challenges and solutions. Clin Cosmet Investig Dermatol. 2016;9(9):473-82.

9. Villar RR, Fernández SP, Garea CC, Pillado MT, Barreiro VB, Martín CG. Quality of life and anxiety in women with breast cancer before and after treatment. Rev Lat Am Enfermagem. 2017;25:e2958.

10. Sawada NO, Nicolussi AC, de Paula JM, Garcia-Caro MP, Marti-Garcia C, Cruz-Quintana F. Quality of life of Brazilian and Spanish cancer patients undergoing chemotherapy: an integrative literature review. Rev Lat Am Enfermagem. 2016;24:e2688.

11. WHO. The World Health Organization Quality of Life assessment (WHOQOL): position paper from the World Health Organization. Soc Sci Med. 1995;41(10):1403-9.
12. Radiation Therapy Oncology Group (RTOG). Acute Radiation Morbidity Scoring Criteria. RTOG:C2020. [cited 2020 Jul 16]. Available from: https://www.rtog.org/researchassociates/adverseeventreporting/ rtogeortclateradiationmorbidityscoringschema.aspx

13. Fayers P, Bottomley A; EORTC Quality of Life Group; Quality of Life Unit; European Organisation for Research and Treatment of Cancer. Quality of life research within the EORTC-the EORTC QLQ-C30. Eur J Cancer. 2002;38(4 Suppl 4):S125-33.

14. Fayers PM, Aaronson NK, Bjordal K, Groenvold M, Curran D, Bottomley A, et al. The EORTC QLQ-C30 scoring manual. 3rd ed. Brussels; 2001.

15. Cui Z, Xin M, Yin H, Zhang J, Han F. Topical use of olive oil preparation to prevent radiodermatitis: results of a prospective study in nasopharyngeal carcinoma patients. Int J Clin Exp Med. 2015;8(7):11000-6.

16. Cardozo AS, Simões FV, Santos V0, Portela LF, Silva RC. Severe radiodermatitis and risk factors associated in head and neck cancer patients. Texto Contexto Enferm. 2020;9:e20180343.

17. Ferreira EB, Ciol MA, Vasques Cl, Bontempo PS, Vieira NN, Silva LF, et al. Gel of chamomile vs. urea cream to prevent acute radiation dermatitis in patients with head and neck cancer: a randomized controlled trial. J Adv Nurs. 2016;72(8):1926-34.

18. Rao S, Hegde SK, Baliga-Rao MP, Palatty PL, George T, Baliga MS. An aloe vera-based cosmeceutical cream delays and mitigates ionizing radiation-induced dermatitis in head and neck cancer patients undergoing curative radiotherapy: a clinical study. Medicines (Basel). 2017;4(3):44.

19. Stylianou C, Kafasi A, Papageorgou DK. Nurse led interventions for patients with head and neck cancer. Nosileftiki. 2017;56(2):97-106.

20. Freire ME, Costa SF, Lima RA, Sawada NO. Health-related quality of life of patients with cancer in palliative care. Texto Contexto Enferm. 2018;27(2):e5420016.

21. Dean LT, Moss SL, Ransome Y, Frasso-Jaramillo L, Zhang Y, Visvanathan $K$, et al. "It still affects our economic situation": long-term economic burden of breast cancer and lymphedema. Support Care Cancer. 2019;27(5):1697-708.

22. Sadovsky AD, Poton WL, Reis-Santos B, Barcelos MR, Silva IC. Índice de Desenvolvimento Humano e prevenção secundária de câncer de mama e colo do útero: um estudo ecológico. Cad Saude Publica. 2015;31(7):1539-50.

23. O'Donovan A, Coleman M, Harris R, Herst P. Prophylaxis and management of acute radiation-induced skin toxicity: a survey of practice across Europe and the USA. Eur J Cancer Care (Engl). 2015;24(3):425-35.

24. Manas A, Santolaya M, Ciapa VM, Belinchón B, Tully F. Topical R1 and $\mathrm{R} 2$ prophylactic treatment of acute radiation dermatitis in squamous cell carcinoma of the head and neck and breast cancer patients treated with chemoradiotherapy. Eplasty. 2015;15:e25.

25. Elliott EA, Wright JR, Swann RS, Nguyen-Tân F, Takita C, Bucci MK, et al.; Radiation Therapy Oncology Group Trial 99-13. Phase III Trial of an emulsion containing trolamine for the prevention of radiation dermatitis in patients with advanced squamous cell carcinoma of the head and neck: results of Radiation Therapy Oncology Group Trial 99-13. J Clin Oncol. 2006;24(13):2092-7.

26. Wells M, Macmillan M, Raab G, MacBride S, Bell N, MacKinnon K, et al. Does aqueous or sucralfate cream affect the severity of erythematous radiation skin reactions? A randomised controlled trial. Radiother Oncol. 2004;73(2):153-62.

27. Hindley A, Zain Z, Wood L, Whitehead A, Sanneh A, Barber D, et al. Mometasone furoate cream reduces acute radiation dermatitis in 
patients receiving breast radiation therapy: results of a randomized trial. Int J Radiat Oncol Biol Phys. 2014;90(4):748-55.

28. Silva AM, Mambrini JV, Souza JP, Andrade FB, Costa MF. Hospitalizations among older adults: results from ELSI-Brazil. Rev Saude Publica. 2018;52(2):1-10.

29. Minosso JS, Souza LJ, Oliveira MA. Rehabilitation in palliative care. Texto Contexto Enferm. 2016;25(3):e1470015.

30. Jagannathan A, Juvva S. Emotions and coping of patients with head and neck cancers after diagnosis: A qualitative content analysis. J Postgrad Med. 2016;62(3):143-9.

31. Bazire L, Fromantin I, Diallo A, de la Lande B, Pernin V, Dendale R, et al. Hydrosorb ${ }^{\circledR}$ versus control (water based spray) in the management of radio-induced skin toxicity: results of multicentre controlled randomized trial. Radiother Oncol. 2015;117(2):229-33.
32. Chan RJ, Mann J, Tripcony L, Keller J, Cheuk R, Blades R, et al. Natural oil-based emulsion containing allantoin versus aqueous cream for managing radiation-induced skin reactions in patients with cancer: a phase 3, double-blind, randomized, controlled trial. Int J Radiat Oncol Biol Phys. 2014;90(4):756-64.

33. Andrade FS, Clark RM, Ferreira ML. Effects of low-level laser therapy on wound healing. Rev Col Bras Cir. 2014;41(2):129-33.

34. Borges JA, Quintão MM, Chermont SM, Filho HT, Mesquita ET. Fadiga: Um Sintoma Complexo e seu Impacto no Câncer e na Insuficiência Cardíaca. Int J Cardiovasc Sci. 2018;31(4):433-42.

35. Younus J, Lock M, Vujovic 0, Yu E, Malec J, D'Souza D, et al. A casecontrol, mono-center, open-label, pilot study to evaluate the feasibility of therapeutic touch in preventing radiation dermatitis in women with breast cancer receiving adjuvant radiation therapy. Complement Ther Med. 2015;23(4):612-6. 